\title{
Síntesis, caracterización y actividad fotocatalítica de óxido de titanio modificado con nitrógeno
}

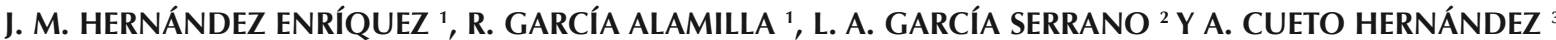 \\ ${ }^{1}$ Instituto Tecnológico de Cd. Madero, División de Estudios de Posgrado e Investigación, Juventino Rosas y Jesús Urueta s/n, \\ Col. Los Mangos, 89440 Cd. Madero, Tamaulipas, México. \\ ${ }^{2}$ Instituto Politécnico Nacional, Escuela Superior de Ingeniería Textil, Av. Instituto Politécnico Nacional s/n, Edificio \#8, \\ Col. Linda Vista, Delegación Gustavo A. Madero, 07738 México D.F., México. \\ ${ }^{3}$ Universidad Autónoma Metropolitana - Azcapotzalco, Av. San Pablo \#180, Col. Reynosa Tamaulipas, \\ Delegación Azcapotzalco, 02200 México D.F., México.
}

\begin{abstract}
Se sintetizaron óxidos de titanio $\left(\mathrm{TiO}_{2}\right)$ a partir de la precipitación del tetracloruro de titanio $\left(\mathrm{TiCl}_{4}\right)$ con hidróxido de amonio $\left(\mathrm{NH}_{4} \mathrm{OH}\right)$. Los materiales sintetizados se caracterizaron mediante fisisorción de nitrógeno, difracción de rayos X, espectroscopía infrarroja, reflectancia difusa U.V.-visible y la actividad fotocatalítica de los mismos se evaluó en la degradación del naranja de metilo. Mediante el método de síntesis se logró dopar la estructura del óxido de titanio con nitrógeno $\left(\mathrm{N}-\mathrm{TiO}_{2}\right)$, estabilizando la fase anatasa y obteniéndose materiales catalíticos mesoporosos y nanocristalinos. El óxido de titanio de mayor área específica $\left(132 \mathrm{~m}^{2} / \mathrm{g}\right)$ degradó el azo-colorante al $100 \%$ en 180 minutos de reacción.
\end{abstract}

Palabras clave: síntesis, óxidos de titanio, materiales mesoporosos, materiales nanocristalinos, azo-compuesto

Synthesis, characterization and photocatalytic activity of titanium oxide modified with nitrogen

Titanium oxides $\left(\mathrm{TiO}_{2}\right)$ were synthesized by precipitation of titanium tetrachloride $\left(\mathrm{TiCl}_{4}\right)$ using ammonium hydroxide $\left(\mathrm{NH}_{4} \mathrm{OH}\right)$. The synthesized materials were characterized by means of nitrogen physisorption, X-ray diffraction, infrared spectroscopy, U.V.-visible diffuse reflectance spectroscopy and the photocatalytic activity of the samples were measured by the degradation of the methyl orange. By means of this synthesis method we have doped the titanium oxide structure with nitrogen $\left(\mathrm{N}-\mathrm{TiO}_{2}\right)$, stabilizing the anatase phase and obtaining mesoporous and nanocrystalline materials. The titanium oxide with higher specific surface area $\left(132 \mathrm{~m}^{2} / \mathrm{g}\right)$ degraded the azo-compound to $100 \%$ in 180 min of reaction.

Keywords: synthesis, titanium oxides, mesoporous materials, nanocrystalline materials, azo-compound

\section{INTRODUCCIÓN}

Los efluentes de la industria textil presentan un gran potencial de impacto sobre el medio ambiente, debido a los grandes volúmenes de agua residual generados con un alto contenido de materia orgánica y una fuerte coloración. A causa de la gran estabilidad de los colorantes (azocompuestos), los procesos convencionales para el tratamiento de aguas residuales tales como adsorción, floculación y procesos de lodos activados no son eficientes para la decoloración de los efluentes textileros, ya que no pueden degradar eficientemente los compuestos orgánicos o generan contaminantes secundarios que pueden ser tóxicos para el medio ambiente y que requieren de un tratamiento adicional (1-3). Una alternativa al tratamiento convencional de las aguas residuales es la oxidación fotocatalítica (fotocatálisis), la cual, ha despertado un gran interés debido a que las investigaciones han mostrado la degradación de contaminantes orgánicos a bajas y medianas concentraciones, con una generación baja en contaminantes secundarios. La fotocatálisis heterogénea consiste en la degradación del contaminante a través de la utilización de catalizadores (óxidos semiconductores), radiación ultravioleta y/o solar, generando con ello radicales
$\left(\mathrm{O}_{2} \bullet-, \mathrm{HO}_{2} \bullet \mathrm{y} / \mathrm{O} \mathrm{OH} \bullet\right)$ los cuales son responsables de la oxidación de dichos contaminantes $(4,5)$.

El óxido de titanio $\left(\mathrm{TiO}_{2}\right)$ es uno de los semiconductores del tipo cerámico más investigado dentro de los procesos de oxidación avanzada, debido a que éste posee propiedades fotoconductoras y fotocatalíticas que lo presentan como uno de los óxidos de mayor impacto en la degradación de azo-colorantes, oxidación de compuestos orgánicos volátiles (COV's) y degradación de compuestos orgánicos clorados, entre otras (6-8). Además de sus propiedades fotoconductoras, sus características texturales y estructurales, en conjunto con su cristalinidad, desempeñan un papel importante dentro de cualquier proceso catalítico $(9,10)$. Aparentemente la eficiencia de las reacciones fotocatalíticas son inducidas por una deseable área específica y en el caso del $\mathrm{TiO}_{2}$ por su fase anatasa estabilizada (11). Se especula que soportes mesoporosos del tipo $\mathrm{TiO}_{2}$ pueden ser candidatos prometedores para el campo de la fotocatálisis debido a su alta área específica y a la conveniente transferencia de masa que pudiera darse dentro de sus mesoporos. Sin embargo, estos materiales son comúnmente preparados mediante el 
uso de surfactantes tipo plantilla (compuestos orgánicos de cadena larga), los cuales, al ser eliminados por tratamiento térmico, causan el colapsamiento de la estructura porosa del material en la mayoría de los casos (12). Por estas razones, en el presente trabajo se sintetizó $\mathrm{TiO}_{2}$ modificado con nitrógeno (N) mediante la precipitación del tetracloruro de titanio $\left(\mathrm{TiCl}_{4}\right)$ a baja temperatura $\left(50^{\circ} \mathrm{C}\right)$, en presencia de ácido acético glacial, utilizando hidróxido de amonio $\left(\mathrm{NH}_{4} \mathrm{OH}\right)$ como plantilla modificadora, en lugar de una plantilla orgánica de cadena larga. La actividad fotocatalítica del $\mathrm{TiO}_{2}$ sintetizado se evaluó en la degradación del naranja de metilo, elegido como molécula modelo por ser uno de los constituyentes de los desechos de la industria textil.

\section{PROCEDIMIENTO EXPERIMENTAL}

\subsection{Síntesis de óxidos de titanio $\left(\mathrm{N}-\mathrm{TiO}_{2}\right)$}

Para sintetizar $\mathrm{N}-\mathrm{TiO}_{2^{\prime}} 12.5 \mathrm{ml}$ de una solución acuosa de $\mathrm{TiCl}_{4} 3 \mathrm{M}$, se adicionaron mediante goteo lento en 75 $\mathrm{ml}$ de agua desionizada, previamente enfriada a $3^{\circ} \mathrm{C}$ para evitar la drástica hidrólisis del $\mathrm{TiCl}_{4}\left(\mathrm{TiCl}_{4^{\prime}} \mathrm{J}\right.$. T. Baker, grado analítico). La solución fue homogenizada mediante agitación suave y calentada a $50^{\circ} \mathrm{C}$. Después, ácido acético glacial $\left(\mathrm{CH}_{3} \mathrm{COOH}\right.$, Fermont, 99.8\%) y una solución de $\mathrm{NH}_{4} \mathrm{OH}$ al $30 \%$ peso $\left(\mathrm{NH}_{4} \mathrm{OH}\right.$, Fermont, $30 \%$ ), se adicionaron mediante goteo lento y en agitación vigorosa hasta alcanzar un $\mathrm{pH}=$ 8. El precipitado se añejó en la solución madre durante 72 $\mathrm{h}$ y después se filtró y lavó con agua desionizada y alcohol etílico $\left(\mathrm{CH}_{3} \mathrm{CH}_{2} \mathrm{OH}\right.$, Fermont, $\left.99.5 \%\right)$ hasta alcanzar un $\mathrm{pH}=$ 7. El N-TiO ${ }_{2}$ se obtuvo después de un secado a $70^{\circ} \mathrm{C}$ durante $24 \mathrm{~h}$, seguido de una calcinación en flujo de aire extraseco a $450^{\circ} \mathrm{C}$ por $3 \mathrm{~h}$. Durante la síntesis se varió la cantidad de ácido acético para analizar el efecto de éste parámetro sobre las propiedades fisicoquímicas del material catalítico. La nomenclatura de los materiales sintetizados acorde a los parámetros experimentales manejados en este trabajo, se puede observar en la Tabla I.

\subsection{Caracterización de los óxidos de titanio $\left(\mathrm{N}-\mathrm{TiO}_{2}\right)$}

La fisisorción denitrógeno para determinar elárea específica de los materiales se efectuó a la temperatura del nitrógeno líquido $\left(-196^{\circ} \mathrm{C}\right)$ en un equipo marca Quantachrome modelo Autosorb-1. Previo al análisis, las muestras se desgasificaron durante $2 \mathrm{~h}$ a $350^{\circ} \mathrm{C}$. Los patrones de difracción de rayos $\mathrm{X}$ se obtuvieron en un Difractómetro D800-Advance Bruker AXS el

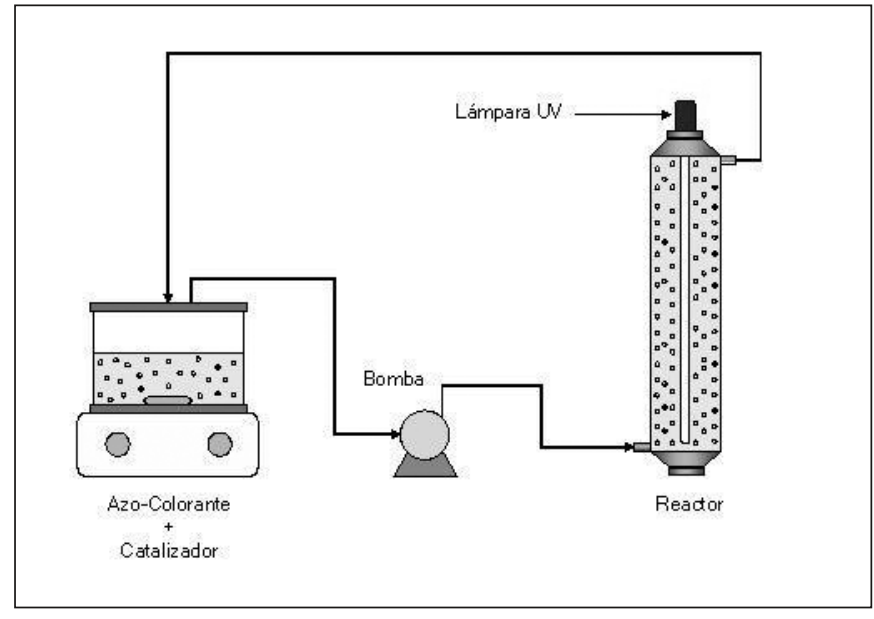

Fig. 1- Esquema del equipo experimental utilizado en la degradación del naranja de metilo con luz U.V.

cual utilizó radiación Cu K $\alpha(\lambda=1.5406$ Å) y un monocromador de grafito en el haz secundario; las intensidades de las líneas de difracción se obtuvieron en el intervalo de $0-80^{\circ}$ en la escala $2 \theta$, con pasos de $0.02^{\circ}$ y $2.4 \mathrm{~s}$ por punto. La espectroscopía de infrarrojo se realizó en un espectrómetro de transformada de Fourier marca Perkin-Elmer modelo Spectrum One con pastillas transparentes conteniendo la muestra a analizar y $\mathrm{KBr}$ como aglutinante ( $90 \%$ en peso), utilizando un número de barridos de 16 y una resolución de $4 \mathrm{~cm}^{-1}$. Los espectros de reflectancia difusa U.V.-visible se obtuvieron mediante un espectrofotómetro Shimadzu UV-2401, utilizando $\mathrm{BaSO}_{4}$ como muestra de referencia y realizando el análisis de las muestras en el intervalo de $200-800 \mathrm{~nm}$.

\subsection{Actividad fotocatalítica}

La actividad fotocatalítica se evaluó en la degradación del naranja de metilo $\left(\left[\left(\mathrm{CH}_{3}\right)_{2} \mathrm{NC}_{6} \mathrm{H}_{4} \mathrm{~N}=\mathrm{NC}_{6} \mathrm{H}_{4} \mathrm{SO}_{3} \mathrm{Na}\right]\right.$, Fermont, grado analítico) cuya concentración inicial se estableció en $20 \mathrm{mg} / \mathrm{L}$. La reacción se llevó a cabo en un reactor cilíndrico tipo semi-batch con recirculación, situando en el centro del mismo, una lámpara tubular de mercurio con una potencia de $150 \mathrm{~W}$ utilizada como fuente de radiación ultravioleta. El fotocatalizador $(0.5 \mathrm{~g})$ se suspendió mediante agitación magnética en la solución problema ( 1 L) dentro de un contenedor y ambos fueron alimentados al reactor mediante una bomba a razón de $1.5 \mathrm{~L} / \mathrm{min}$ (Figura 1). La concentración del colorante en función del tiempo de reacción se analizó

TABLA I. NOMENCLATURA DE LOS MATERIALES SINTETIZADOS.

\begin{tabular}{|c|c|c|c|}
\hline Material & Temperatura de calcinación $\left({ }^{\circ} \mathrm{C}\right)$ & Ácido acético $(\mathrm{ml})$ & Descripción del material \\
\hline $\mathrm{TiO}_{2}$ Degussa P-25 & --- & -- & Óxido de titanio comercial \\
\hline $\mathrm{MA}\left[\mathrm{Ti}(\mathrm{OH})_{4}\right]$ & 70 & 2.5 & Hidróxido de titanio \\
\hline $\mathrm{MB}\left[\mathrm{TiO}_{2}\right]$ & 450 & 2.5 & Oxido de titanio \\
\hline $\mathrm{MC}\left[\mathrm{TiO}_{2}\right]$ & 450 & 1.25 & Óxido de titanio \\
\hline
\end{tabular}




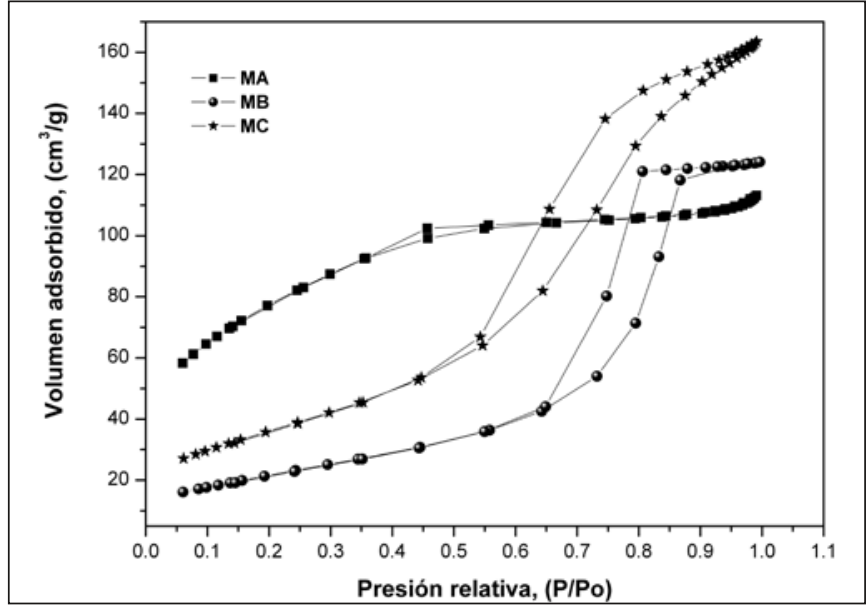

Fig. 2- Isotermas de adsorción-desorción de nitrógeno obtenidas con los óxidos de titanio sintetizados.

en un espectrofotómetro U.V.-visible (Spectronic 21D, Milton Roy). El catalizador se separó de la alícuota de reacción mediante membranas Millipore de acetato de celulosa con diámetro de poro de $0.22 \mu \mathrm{m}$.

\section{RESULTADOS Y DISCUSIÓN}

\subsection{Fisisorción de nitrógeno}

Las propiedades texturales de los óxidos de titanio sintetizados se presentan en la Tabla II. Como puede observarse en esta tabla, el hidróxido de titanio (material MA) reduce su área específica 3.5 veces y aumenta hasta 2.5 veces su diámetro de poro promedio cuando éste se somete a un tratamiento térmico a $450^{\circ} \mathrm{C}$ durante $3 \mathrm{~h}$, esto ocurre como resultado de la sinterización del material durante el proceso de calcinación. Las áreas específicas de los óxidos de titanio están influenciadas por la cantidad de ácido utilizado durante la síntesis, presentando el valor más alto de área específica el material MC (132 $\left.\mathrm{m}^{2} / \mathrm{g}\right)$, el cual fue sintetizado con el menor volumen de ácido acético. En general, la fisisorción de nitrógeno mostró áreas específicas comprendidas entre $79-283 \mathrm{~m}^{2} / \mathrm{g}$, valores que superan el área específica del óxido de titanio comercial Degussa P-25 $\left(51 \mathrm{~m}^{2} / \mathrm{g}\right)$. La Figura 2 muestra que las isotermas de adsorción-desorción obtenidas para los materiales calcinados (materiales $\mathrm{MB}$ y $\mathrm{MC}$ ) son del tipo IV, características de materiales mesoporosos. Su

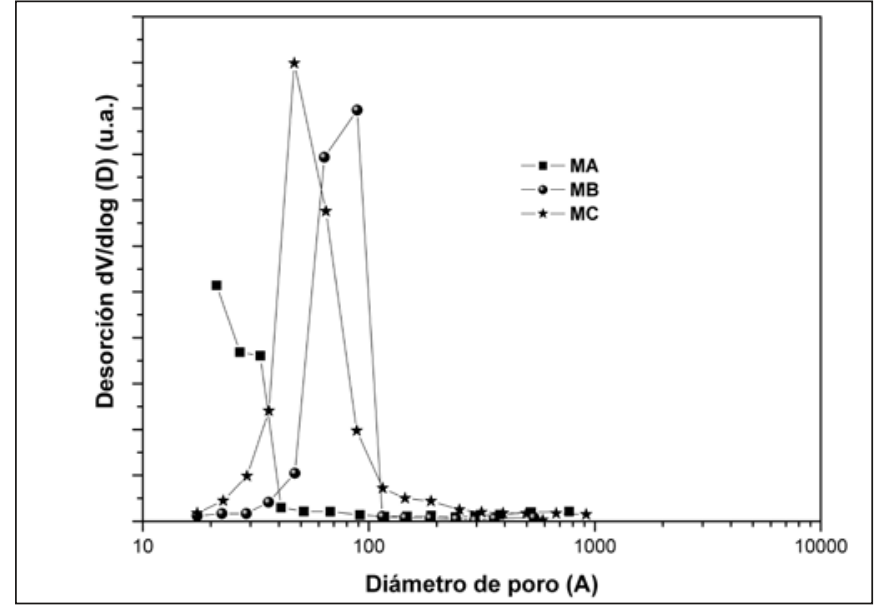

Fig. 3- Distribución del tamaño de poro en los óxidos de titanio sintetizados.

isoterma de desorción forma un amplio ciclo de histéresis tipo II. En contraste, el hidróxido de titanio (material MA) presentó una isoterma tipo I característica de materiales microporosos con un fino ciclo de histéresis que implica una estrecha distribución de tamaño de poro $(13,14)$. Por otro lado, la distribución porosa muestra un perfil muy homogéneo con máximos en la frontera de los materiales micro-mesoporosos, la cual se desplaza hacia la región mesoporosa en función de la temperatura de calcinación (Figura 3).

\subsection{Difracción de rayos $X$}

La Figura 4 muestra los patrones de difracción de rayos $\mathrm{X}$ del óxido de titanio comercial $\left(\mathrm{TiO}_{2}\right.$ Degussa P-25) y los óxidos de titanio sintetizados. Los materiales calcinados a $450^{\circ} \mathrm{C}$ (materiales $\mathrm{MB}$ y $\mathrm{MC}$ ) exhibieron líneas de difracción en $2 \theta=25^{\circ}, 37^{\circ}, 48^{\circ}, 54^{\circ}, 55^{\circ}, 62^{\circ}, 71^{\circ}$ y $75^{\circ}$, que corresponden a los planos cristalográficos (101), (004), (200), (105), (211), (204), (116) y (311), que caracterizan la fase anatasa (tetragonal) del óxido de titanio (15). Los tamaños de cristal obtenidos para estos materiales fueron de 15 y $10 \mathrm{~nm}$, respectivamente, clasificándose como sólidos nanocristalinos. El patrón de difracción de rayos X del material MA (hidróxido de titanio) lo muestra como un material completamente amorfo y con pobre cristalinidad. A diferencia de los materiales sintetizados, el óxido de titanio comercial presenta una mezcla de fases cristalinas, una minoritaria perteneciente a la fase rutilo $(2 \theta=$ $27^{\circ}, 36^{\circ}, 41^{\circ}, 44^{\circ}$ y $\left.57^{\circ}\right)$ y otra mayoritaria perteneciente a la fase

TABLA II. PROPIEDADES TEXTURALES DE LOS ÓXIDOS DE TITANIO SINTETIZADOS.

\begin{tabular}{|c|c|c|c|c|c|}
\hline Material & $\begin{array}{c}\text { Temperatura de } \\
\text { calcinación }\left({ }^{\circ} \mathbf{C}\right)\end{array}$ & $\begin{array}{c}\text { Ácido acético } \\
(\mathbf{m l})\end{array}$ & $\begin{array}{c}\text { Área específica } \\
\left(\mathbf{m}^{2} / \mathbf{g}\right)\end{array}$ & $\begin{array}{c}\text { Diámetro } \\
\text { de poro }(\AA)\end{array}$ & $\begin{array}{c}\text { Volumen } \\
\mathbf{d e ~} \mathbf{p o r o}\left(\mathbf{c m}^{3} / \mathbf{g}\right)\end{array}$ \\
\hline $\mathrm{TiO}_{2}$ Degussa P-25 & --- & --- & 51 & 160 & 0.16 \\
\hline $\mathrm{MA}\left[\mathrm{Ti}(\mathrm{OH})_{4}\right]$ & 70 & 2.5 & 283 & 28 & 0.17 \\
\hline $\mathrm{MB}\left[\mathrm{TiO}_{2}\right]$ & 450 & 2.5 & 79 & 56 & 0.19 \\
\hline $\mathrm{MC}\left[\mathrm{TiO}_{2}\right]$ & 450 & 1.25 & 132 & 52 \\
\hline
\end{tabular}


anatasa (tetragonal), observando un tamaño de cristal de 21 $\mathrm{nm}$ (16). Los resultados sugieren que el dopaje de la estructura del óxido de titanio con átomos de nitrógeno provenientes del $\mathrm{NH}_{4} \mathrm{OH}$, estabiliza la fase anatasa del mismo, impidiendo la drástica sinterización del material y el crecimiento abrupto del cristal.

\subsection{Espectroscopía infrarroja}

En la Figura 5 se muestran los espectros de infrarrojo de los óxidos de titanio sintetizados así como también el obtenido con el material comercial Degussa P-25. Todos los espectros muestran señales que aparecen alrededor de los 3400 y 1630 $\mathrm{cm}^{-1}$ las cuales son atribuidas a tensiones vibracionales de grupos -OH y a vibraciones de deformación de tipo tijera de los protones del agua adsorbida en la superficie del $\mathrm{TiO}_{2}[2,17-$ 19]. Las bandas que aparecen en la región de baja frecuencia en los espectros de infrarrojo situadas en el intervalo de 650$450 \mathrm{~cm}^{-1}$ son características de enlaces Ti-O-Ti [20]. Los picos a 2927 y $2854 \mathrm{~cm}^{-1}$ observados en los espectros de los materiales sintetizados, pueden ser asignados a tensiones C-H e indicar la presencia de materia orgánica remante en los sólidos, relacionada con el ácido acético utilizado durante la síntesis $[17,21]$. Las señales que aparecen en el espectro del hidróxido de titanio (material MA) centradas a 3150 y $1401 \mathrm{~cm}^{-1}$ pueden resultar de modos vibracionales del tipo tensión y flexión características de enlaces $\mathrm{N}-\mathrm{H}$, mismas que pueden estar

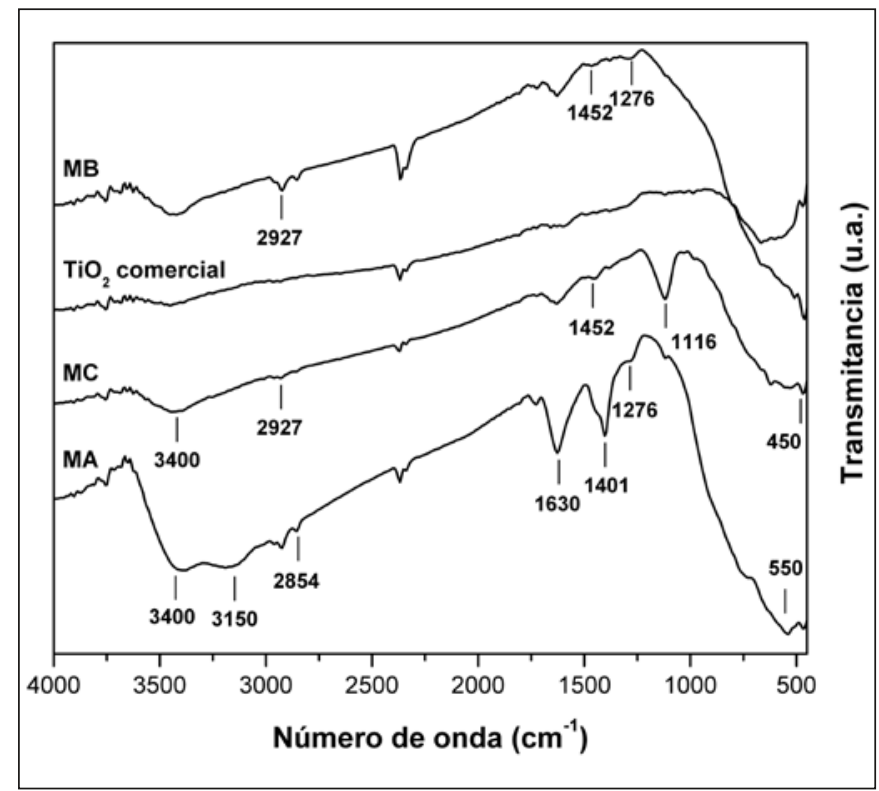

Fig. 5- Espectros de infrarrojo obtenidos con el óxido de titanio comercial $\left(\mathrm{TiO}_{2}\right.$ Degussa P-25) y los óxidos de titanio sintetizados.
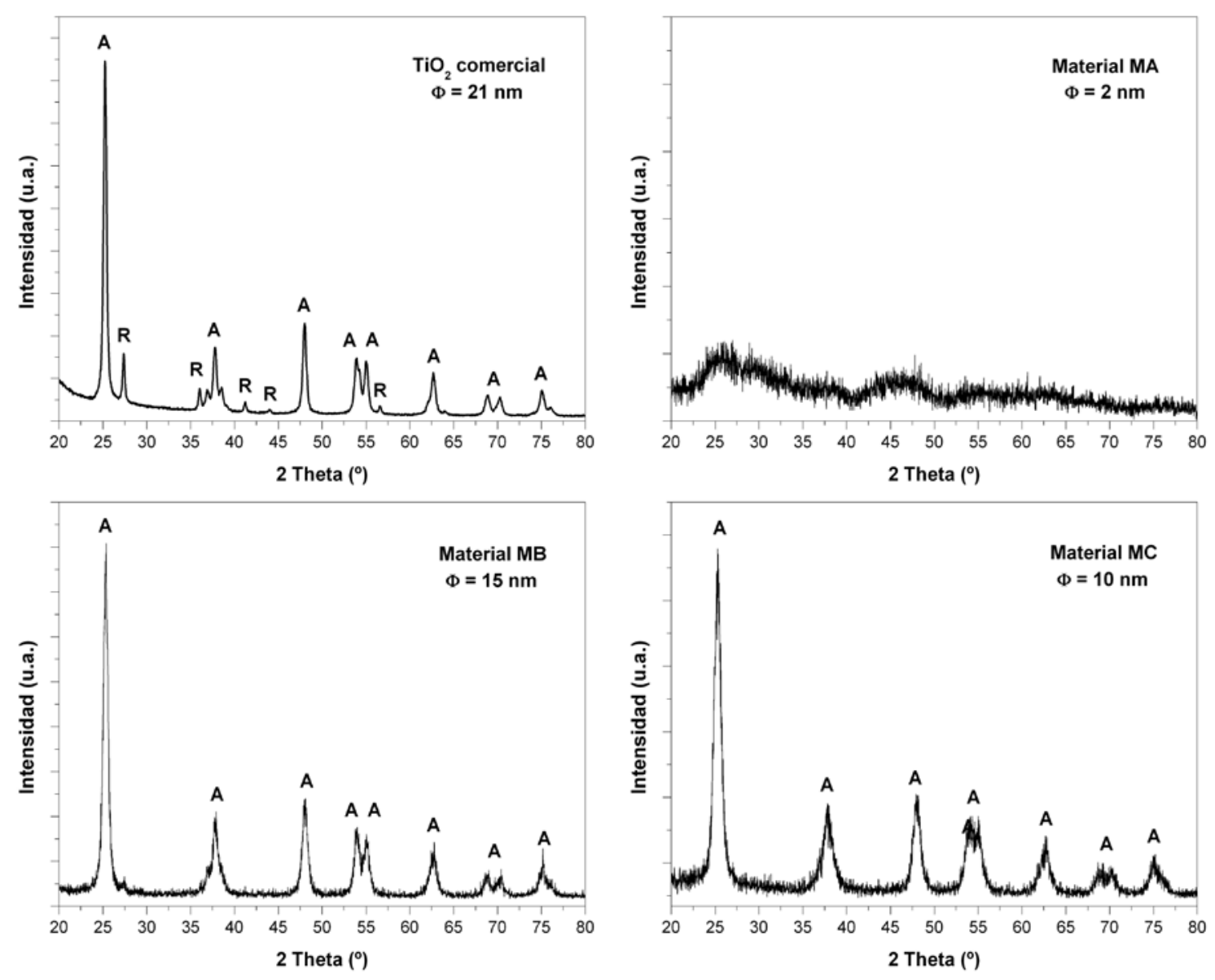

Fig. 4- Diagramas de difracción de rayos $\mathrm{X}$ obtenidos con $\mathrm{TiO}_{2}$ comercial (Degussa P-25) y TiO 2 sintetizado. 
relacionadas con iones amonio $\left(\mathrm{NH}_{4}^{+}\right)$formados durante la disociación del $\mathrm{NH}_{4} \mathrm{OH}$, especies que se encuentran adsorbidas en la superficie del material a bajas temperaturas y que actúan como fuente de nitrógeno, el cual se incorpora a la estructura del $\mathrm{TiO}_{2}$ durante el proceso de calcinación [17,22,23]. Los espectros de infrarrojo de los materiales sintetizados, también muestran bandas a 1452, 1276 y $1116 \mathrm{~cm}^{-1}$ las cuales podrían ser atribuidas a la presencia de especies nitrogenadas tales como $\mathrm{NO}_{3}^{-}, \mathrm{NO}_{2}^{-}$y/o $\left(\mathrm{N}_{2} \mathrm{O}_{2}\right)^{2-}[17,24]$. Debido a que el intervalo de frecuencia de absorción de los $\mathrm{NO}_{3}^{-}$y $\mathrm{NO}_{2}^{-}\left(1450-1250 \mathrm{~cm}^{-1}\right)$ se solapa, no es posible señalar con exactitud que especie nitrogenada esta presente en el material. El pico centrado a $1116 \mathrm{~cm}^{-1}$ ha sido atribuido en otros trabajos a la formación de un anión hiponitrito $\left(\left(\mathrm{N}_{2} \mathrm{O}_{2}\right)^{2-}\right)$, corroborando su presencia por XPS y sugiriendo que este anión identifica la presencia de nitrógeno estructural en la red del óxido de titanio [25-27]. Aunado a esto, las bandas situadas a 1452 y $1250 \mathrm{~cm}^{-1}$ también podrían indicar la nitruración del material.

\subsection{Reflectancia difusa U.V.-visible}

La Figura 6 muestra los espectros de reflectancia difusa U.V.-visible del óxido de titanio comercial y los óxidos de titanio sintetizados. En esta figura es posible observar que todos los materiales catalíticos presentan una fuerte absorción de luz en longitudes de onda alrededor de los $400 \mathrm{~nm}$, la cual es atribuida a las transiciones de los electrones de la banda de valencia a la banda de conducción pertenecientes al $\mathrm{TiO}_{2}$. Sin embargo, con la incorporación de especies de nitrógeno en la estructura del óxido de titanio y la utilización de ácido acético glacial durante la síntesis, se presentan ligeras diferencias entre los materiales. Se puede observar que cuando se utiliza mayor cantidad de ácido acético, menor cantidad de especies de nitrógeno quedan retenidas en la estructura del material después del proceso de calcinación (Figura 5), reflejándose en una mayor absorción de luz en el material MB (Figura 6). Las especies de nitrógeno retenidas en los óxidos de titanio confieren a los mismos una ligera coloración amarillenta, la cual se intensifica para el material MC causando una menor absorción de luz. Probablemente esta sea la razón por la cual el óxido de titanio comercial (polvo blanco) presenta una longitud de onda mayor en comparación con los óxidos de titanio sintetizados. Acorde con los resultados de Wawrzyniak y Morawski (2), la disminución de la reflectancia es causada por el cambio de color obtenido en los materiales catalíticos, el cual depende de la temperatura de tratamiento térmico y

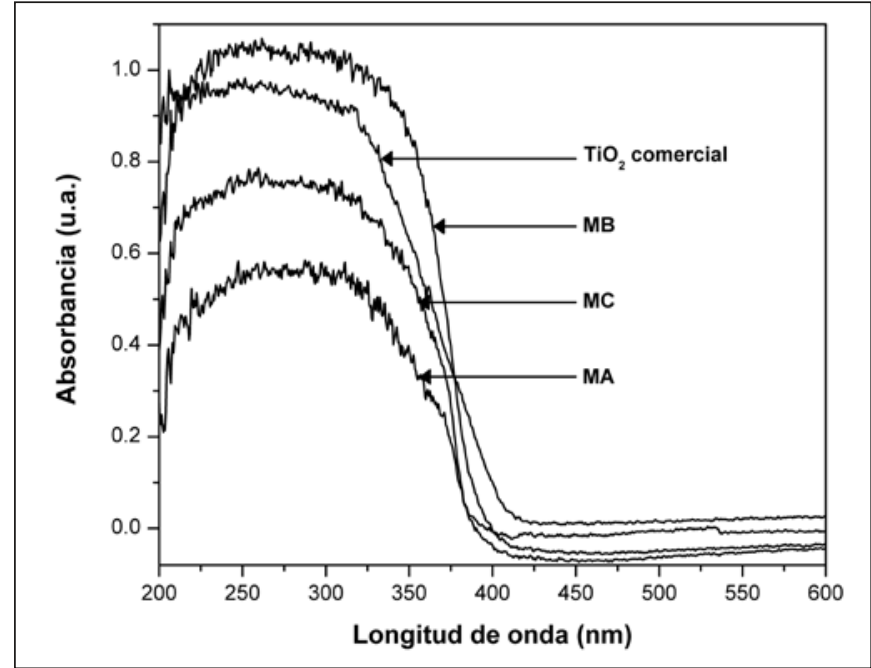

Fig. 6- Espectros de reflectancia difusa U.V.-visible obtenidos con el óxido de titanio comercial $\left(\mathrm{TiO}_{2}\right.$ Degussa P-25) y los óxidos de titanio sintetizados.

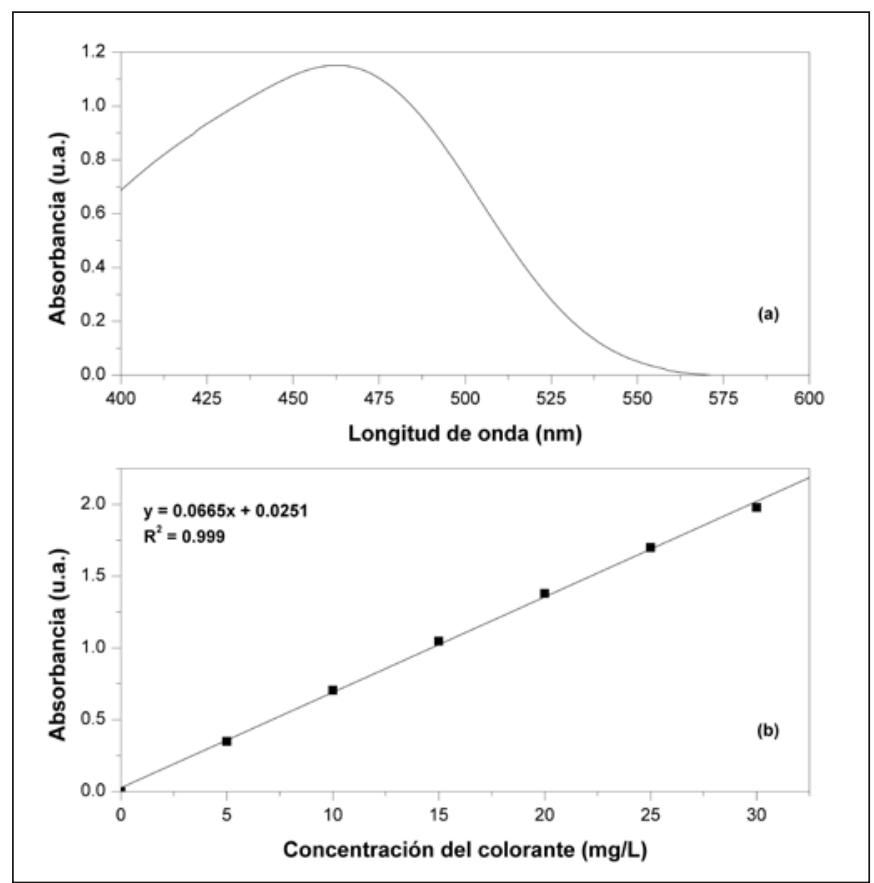

Fig. 7- Espectro U.V.-visible (a) y curva de calibración del naranja de metilo (b).

TABLA III. TAMAÑO DE CRISTAL, LONGITUD DE ONDA Y ENERGÍA DE BANDA PROHIBIDA. PARÁMETROS DETERMINADOS PARA DIFERENTES ÓXIDOS DE TITANIO.

\begin{tabular}{|c|c|c|c|c|}
\hline Material & $\begin{array}{c}\text { Temperatura } \\
\text { de calcinación }\left({ }^{\circ} \mathbf{C}\right)\end{array}$ & $\begin{array}{c}\text { Tamaño } \\
\text { de cristal }(\mathbf{n m})\end{array}$ & $\begin{array}{c}\text { Longitud } \\
\text { de onda }(\mathbf{n m})\end{array}$ & $\begin{array}{c}\text { Energía } \\
\text { de banda }(\mathbf{e V})\end{array}$ \\
\hline $\mathrm{TiO}_{2}$ Degussa P-25 & --- & 21 & 407 & 3.05 \\
\hline $\mathrm{MA}\left[\mathrm{Ti}(\mathrm{OH})_{4}\right]$ & 70 & n.d. & 388 & 3.20 \\
\hline $\mathrm{MB}\left[\mathrm{TiO}_{2}\right]$ & 450 & 15 & 393 & 3.16 \\
\hline $\mathrm{MC}\left[\mathrm{TiO}_{2}\right]$ & 450 & 10 & 391 & 3.17 \\
\hline
\end{tabular}

Tamaño de cristal obtenido por difracción de rayos $\mathrm{X}$

n.d. $=$ no determinado 
cambia de un ligero color amarillento a un amarillo intenso. Acordando también que la tendencia observada en el cambio de color puede ser debido a la concentración de átomos de nitrógeno en las partículas de $\mathrm{TiO}_{2-x} \mathrm{~N}_{x}$.

Los valores de energía de banda prohibida de los óxidos de titanio se estimaron mediante la ecuación [1] (28):

$$
\alpha(h v)=A\left(h v-E_{g}\right)^{m / 2}
$$

Donde $\alpha$ representa el coeficiente de absorción, $h v$ es la energía del fotón, $A$ es una constante y $m=1$ para una transición directa entre la banda de valencia y la banda de conducción. La energía de banda prohibida ( $E_{\mathrm{g}}$ ) se determinó utilizando los espectros de reflectancia difusa U.V.-visible de la Figura 6, mediante la extrapolación de una línea recta a partir de la curva de absorción hacia el eje de la abscisa. Cuando $\alpha$ es igual a cero, entonces la ecuación [1] se reduce a la expresión [2]:

$$
E_{g}=h v=\frac{h c}{\lambda}
$$

En donde la longitud de onda $(\lambda)$ expresada en nanómetros correspondiente a dicha extrapolación, se convierte a unidades de energía en electrón volts $(\mathrm{eV})$. En la Tabla III se puede apreciar un aumento en la energía de banda prohibida en función de la disminución del tamaño de cristal. El óxido de titanio comercial ( $\mathrm{TiO}_{2}$ Degussa P-25) que presenta el tamaño de cristal más grande $(21 \mathrm{~nm})$, comienza a absorber cerca de los $407 \mathrm{~nm}$, longitud de onda que corresponde a una energía de banda prohibida de $3.05 \mathrm{eV}$. Para el caso particular de los óxidos de titanio sintetizados en los cuales el tamaño del cristal disminuye, los materiales comienzan a absorber a longitudes de onda más cortas $(<400 \mathrm{~nm})$ y presentan valores de energía de banda prohibida ligeramente más grandes. Se infiere que con la reducción del tamaño de cristal, el ancho de banda del semiconductor se incrementa, atribuyéndose al efecto del tamaño quántico de la partícula (29).

Las especies de nitrógeno retenidas en la estructura de los óxidos de titanio sintetizados disminuyeron el tamaño de cristal y provocaron un cambio en la coloración de los materiales, reflejándose en un incremento en el ancho de banda del semiconductor.

\subsection{Actividad fotocatalítica}

Una alícuota de la solución del naranja de metilo elegido como molécula modelo para evaluar la fotoactividad de los óxidos de titanio, se analizó en el espectrofotómetro U.V.visible en el intervalo de longitud de onda de 400-600 nm, esto con la finalidad de determinar la máxima absorción del azo-colorante, tal como se muestra en la Figura 7a. La máxima absorción del azo-compuesto establecida a $460 \mathrm{~nm}$ se utilizó para elaborar la curva de calibración mostrada en la Figura 7b, a partir de la cual se determinó el cambio de concentración en la solución del naranja de metilo durante el proceso fotocatalítico.

La Figura 8 presenta la actividad fotocatalítica del óxido de titanio comercial y la de un óxido de titanio sintetizado (material MC) evaluadas bajo las mismas condiciones de reacción. El perfil de actividad fotocatalítica muestra una degradación del azo-colorante de $100 \%$ con ambos materiales, sin embargo, el catalizador comercial ( $\mathrm{TiO}_{2}$ Degussa P-25) logra degradar el azo-compuesto a 90 min de reacción, tornándose la degradación más lenta con el material sintetizado. Esta diferencia en tiempos de reacción puede ser explicada en base a la capacidad de absorción, longitudes de onda y energías de banda prohibida determinadas en ambos materiales. En contraste, solo un pequeño decremento en la concentración de la solución problema pudo observarse cuando ésta era irradiada con luz ultravioleta en ausencia de catalizador. El proceso de fotólisis directa al cual es atribuido éste

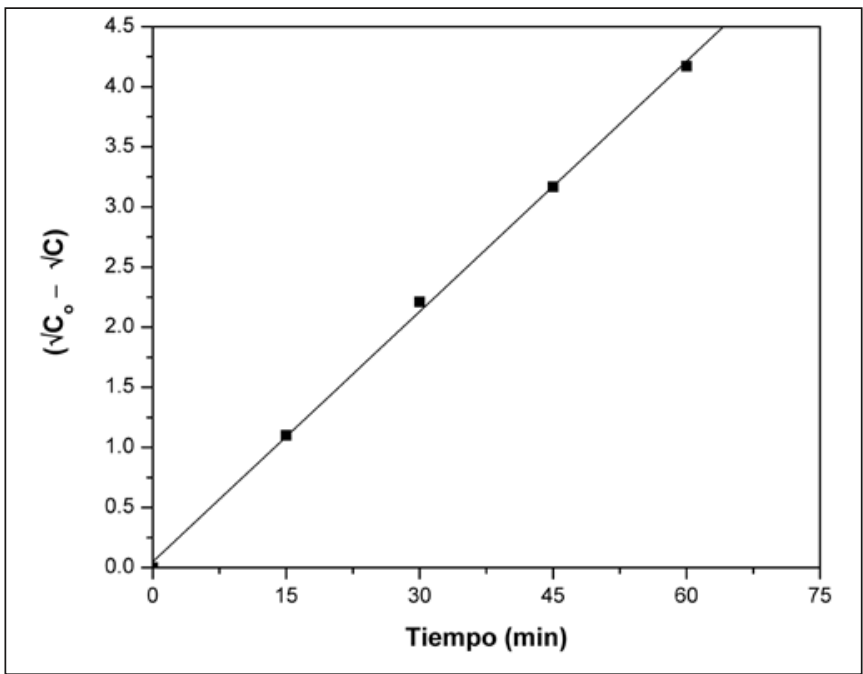

Fig. 9- Ajuste de datos experimentales a una cinética de orden 0.5 desarrollada por el catalizador $\mathrm{TiO}_{2}$ comercial ( $\mathrm{TiO}_{2}$ Degussa P-25) durante la degradación del naranja de metilo. Condiciones de operación: 0.5 $\mathrm{g} / \mathrm{L}$ de $\mathrm{TiO}_{2}$, Luz U.V. (150 W), volumen del azo-colorante $=1 \mathrm{~L}$ y agitación constante.
Fig. 8- Perfil de actividad fotocatalítica desarrollado durante la degradación del naranja de metilo con el catalizador $(\bullet) \mathrm{TiO}_{2}$ comercial $\left(\mathrm{TiO}_{2}\right.$ Degussa P-25), ( $\square) \mathrm{TiO}_{2}$ sintetizado (material MC), sin catalizador $(\square)$, $\mathrm{TiO}_{2}$ comercial ( $\mathrm{TiO}_{2}$ Degussa P-25) sin iluminación (o). Condiciones de operación: $0.5 \mathrm{~g} / \mathrm{L}$ de $\mathrm{TiO}_{2}$, Luz U.V. $(150 \mathrm{~W})$, volumen del azocolorante $=1 \mathrm{~L}$ y agitación constante. 
TABLA IV. PARÁMETROS CINÉTICOS OBTENIDOS EN LA DEGRADACIÓN DEL NARANJA DE METILO.

\begin{tabular}{|l|c|c|c|}
\hline \multicolumn{1}{|c|}{ Material } & Orden de reacción & $k_{\text {apar }}$ & $\mathbf{R}^{2}$ \\
\hline $\mathrm{TiO}_{2}$ (Degussa P-25) & 0.5 & $0.143\left(\frac{m g}{L}\right)^{1 / 2} \mathrm{~min}^{-1}$ & 0.999 \\
\hline $\mathrm{MC}^{\left.-1 \mathrm{TiO}_{2}\right]}$ & 0 & $0.124\left(\frac{m g}{L}\right) \mathrm{min}^{-1}$ & 0.998 \\
\hline
\end{tabular}

decremento, contribuye con menos del 10\% de la degradación total del colorante después de $3 \mathrm{~h}$ de reacción. El experimento realizado en ausencia de luz ultravioleta utilizando como catalizador al óxido de titanio comercial no mostró resultados significativos. Estos dos últimos experimentos demostraron que la degradación del naranja de metilo necesita de la presencia de un fotocatalizador y de luz ultravioleta para llevarse acabo.

Por otro lado, los resultados experimentales indican la existencia de dos regímenes cinéticos durante la fotodegradación del naranja de metilo, dependientes del catalizador usado. Para el caso particular del óxido de titanio comercial ( $\mathrm{TiO}_{2}$ Degussa P-25), la cinética de reacción se ajusta a un orden igual a 0.5 y es descrita por la ecuación [3]:

$$
-r=-\frac{d C}{d t}=k_{\text {apar }} \sqrt{C}
$$

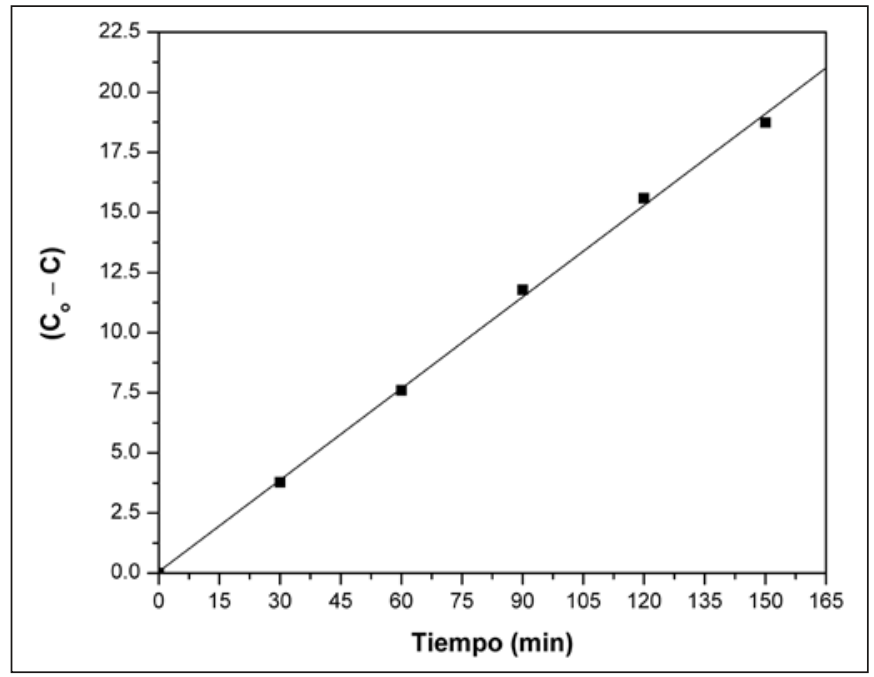

Fig. 10- Ajuste de datos experimentales a una cinética de orden cero desarrollada por el catalizador $\mathrm{TiO}_{2}$ sintetizado (material MC) durante la degradación del naranja de metilo. Condiciones de operación: 0.5 $\mathrm{g} / \mathrm{L}$ de $\mathrm{TiO}_{2}$, Luz U.V. $(150 \mathrm{~W})$, volumen del azo-colorante $=1 \mathrm{~L}$ y agitación constante.
Al integrarse la ecuación [3] con respecto a las condiciones límite: $C=C_{0}$ a $t=0$ y $C=C$ a $t=t$, se obtiene la ecuación [4]:

$$
\sqrt{C_{o}}-\sqrt{C}=\frac{k_{\text {apar }} t}{2}
$$

Donde $C_{o}$ es la concentración inicial del azo-colorante, $C$ la concentración a tiempo $t$ y $k_{\text {apar }}$ representa la constante de velocidad de reacción aparente. Graficando $\left(\sqrt{C_{o}}-\sqrt{C}\right)$ con respecto al tiempo de reacción se obtiene la Figura 9, en la cual se puede observar que los datos experimentales se ajustan a una línea recta, corroborando el modelo cinético propuesto. Vulliet y col., (30) sugieren para éste orden cinético, una reacción con estados disociados del reactante, adsorbidos sobre la superficie del catalizador.

La ecuación 5 describe una cinética de orden cero la cual fue sugerida para la reacción catalizada por el óxido de titanio sintetizado (material MC).

$$
-r=-\frac{d C}{d t}=k_{\text {apar }}
$$

La integración de la ecuación anterior utilizando las condiciones límite establecidas para la ecuación [3], da como resultado la expresión mostrada por la ecuación [6]:

$$
C_{o}-C=k_{\text {apar }} t
$$

Graficando la diferencia de concentraciones $\left(C_{o}-C\right)$ como una función del tiempo de reacción, se puede observar una linealidad obtenida con los datos cinéticos experimentales (Figura 10). El perfil de actividad catalítica mostrado en la Figura 8 para el óxido de titanio sintetizado, se apega a lo mencionado por Levenspiel (31) en relación a que las reacciones son de orden cero solamente en ciertos intervalos de concentración (concentraciones altas). Si la concentración disminuye lo suficiente, suele encontrarse que la velocidad de reacción depende de la concentración, en cuyo caso el orden reacción es superior a cero $(32,33)$.

En la Tabla IV se presentan los valores de las constantes de velocidad de reacción y los coeficientes de correlación para cada perfil de concentración graficados en la Figura 9 y Figura 10, respectivamente. Como se puede apreciar, la constante de 
velocidad de reacción del óxido de titanio comercial es más grande que la del óxido de titanio sintetizado, corroborándose éste hecho con el perfil de actividad fotocatalítica mostrado en la Figura 8 y con la diferencia en tiempos de degradación total.

\section{CONCLUSIONES}

Mediante la precipitación del $\mathrm{TiCl}_{4}$ vía $\mathrm{NH}_{4} \mathrm{OH}$ se logró obtener un óxido de titanio mesoporoso y nanocristalino. El dopaje de la estructura del óxido de titanio con especies de nitrógeno, logró establecer en los materiales sintetizados la fase anatasa, impidió la drástica sinterización y el crecimiento abrupto del cristal, obteniéndose materiales con áreas específicas superiores a la del óxido de titanio comercial $\left(\mathrm{TiO}_{2}\right.$ Degussa P-25). Aunque el óxido de titanio comercial y el óxido de titanio sintetizado degradaron al $100 \%$ el azo-colorante naranja de metilo, el catalizador comercial abatió el tiempo de reacción en 90 min.

\section{BIBLIOGRAFÍA}

(1) T. Velegraki, I. Poulios, M. Charalabaki, N. Kalogerakis, P. Samaras y D. Mantzavinos, «Photocatalytic and sonolytic oxidation of acid orange 7 in aqueous solution», Appl. Catal. B, 62, 159-168 (2006).

(2) B. Wawrzyniak y A.W. Morawski, «Solar-light-induced photocatalytic decomposition of two azo dyes on new $\mathrm{TiO}_{2}$ photocatalyst containing nitrogen», Appl. Catal. B, 62, 150-158, (2006).

(3) A. Pintar, M. Besson y P. Gallezot, «Catalytic wet air oxidation of Kraft bleaching plant effluents in the presence of titania and zirconia supported ruthenium», Appl. Catal. B, 30, 123-139, (2001).

(4) W. Su, J. Chen, L. Wu, X. Wang, X. Wang y X. Fu, «Visible light photocatalysis on praseodymium (III) - nitrate-modified $\mathrm{TiO}_{2}$ prepared by an ultrasound method», Appl. Catal. B, 77, 264-271, (2008).

(5) J. Xing, Z. Shan, K. Li, J. Bian, X. Lin, W. Wang y F. Huang, «Photocatalytic activity of $\mathrm{Nb}_{2} \mathrm{O}_{5} / \mathrm{SrNb}_{2} \mathrm{O}_{6}$ heterojunction on the degradation of methyl orange», J. Phys. Chem. Solids, 69, 23-28, (2008).

(6) N. Bouazza, M.A. Lillo y A. Linares, «Enhancement of the photocatalytic activity of pelletized $\mathrm{TiO}_{2}$ for the oxidation of propane at low concentration», Appl. Catal., B, 77, 284-293, (2008).

(7) A.O. Ibhadon, G.M. Greenway y Y. Yue, «Photocatalytic activity of surface modified $\mathrm{TiO}_{2} / \mathrm{RuO}_{2} / \mathrm{SiO}_{2}$ nanoparticles for azo-dye degradation», Catal. Commun., 9, 153-157, (2008).

(8) D. Fabbri, A. Bianco y E. Pramauro, «Effect of surfactant microstructures on photocatalytic degradation of phenol and chlorophenols», Appl. Catal. B, 62, 21-27, (2006).

(9) S.H. Kang, J.Y. Kim, H.S. Kim y Y.E. Sung, «Formation and mechanistic study of self-ordered $\mathrm{TiO}_{2}$ nanotubes on Ti substrate», J. Ind. Eng. Chem., 14, 52-59, (2008).

(10) M. Addamo, V. Augugliaro, A. Di, E. García, V. Loddo, G. Marci, R. Molinari, L. Palmisano y M. Schiavello, «Preparation, characterization, and photoactivity of polycrystalline nanostructured $\mathrm{TiO}_{2} »$, J. Phys. Chem. B, 108, 3303-3310, (2004).

(11) G. Colón, M.C. Hidalgo y J.A. Navío, «Photocatalytic behaviour of sulphated $\mathrm{TiO}_{2}$ for phenol degradation», Appl. Catal. B, 45, 39-50, (2003).

(12) Q. Sheng, S. Yuan, J. Zhang y F. Chen, «Synthesis of mesoporous titania with high photocatalytic activity by nanocrystalline particle assembly", Microporous Mesoporous Mater., 87, 177-184, (2006).
(13) G. Leofanti, M. Padovan, G. Tozzola y B. Venturelli, «Surface area and pore texture of catalysts», Catal. Today, 41, 207-219, (1998).

(14) S. Storck, H. Bretinger y W.F. Maier, «Characterization of micro- and mesoporous solids by physisorption methods and pore-size analysis», Appl. Catal. A, 174, 137-146, (1998).

(15) P. Weerachawanasak, P. Praserthdam, M. Arai y J. Panpranot, «A comparative study of strong metal-support interaction and catalytic behavior of $\mathrm{Pd}$ catalysts supported on micron- and nano-sized $\mathrm{TiO}_{2}$ in liquid-phase selective hydrogenation of phenylacetylene», J. Mol. Catal. A, 279, 133-139, (2008).

(16) J.R. Sohn y D.Ch. Shin, «Environmentally friendly solid acid catalyst prepared by modifying $\mathrm{TiO}_{2}$ with cerium sulphate for the removal of volatile organic chemicals», Appl. Catal. B, 77, 386-394, (2008).

(17) D.A. Skoog y J.J. Leary, «Espectroscopía de absorción en el infrarrojo», pp. 328 en Análisis instrumental, Mc-Graw Hill, México, (2003).

(18) Y. Huang, Z. Xuxu, Y. Zhongyi, T. Feng, F. Beibei y H. Keshan, «Preparation of nitrogen-doped $\mathrm{TiO}_{2}$ nanoparticle catalyst and its catalytic activity under visible light», Chin. J. Chem. Eng., 15(6), 802-807, (2007).

(19) Y. Zhiyuan y Z. Anning, «Effects of nitrogen doping on microstructure and photocatalytic activity of nanocrystalline $\mathrm{TiO}_{2}$ Powders», J. Wuhan Univ. Technol., 10, 457-461, (2007).

(20) K.H. Yoon, J.S. Noh, Ch.H. Kwon y M. Muhammed, «Photocatalytic behavior of $\mathrm{TiO}_{2}$ thin films prepared by sol-gel process», Mater. Chem. Phys., 95, 79-83, (2006).

(21) K.M.S. Khalil y M.I. Zaki, «Synthesis of high surface area titania via basic hydrolysis of titanium (IV) isopropoxide», Powder Technol., 92, 233-239, (1997).

(22) T. Ihara, M. Miyoshi, Y. Iriyama, O. Matsumoto y S. Sugihara, «Visiblelight-active titanium oxide photocatalyst realized by an oxygen-deficient structure and by nitrogen doping», Appl. Catal. B, 42, 403-409, (2003).

(23) L. Yuexiang, X. Chengfu, P. Shaoquin, L. Gongxuan y L. Shuben, «Eosin Y-sensitized nitrogen-doped $\mathrm{TiO}_{2}$ for efficient visible light photocatalytic hydrogen evolution», J. Mol. Catal. A., 282, 117-123, (2008).

(24) J. Sá y J.A. Anderson, «FT-IR study of aqueous nitrate reduction over Pd/ $\mathrm{TiO}_{2}$, Appl. Catal. B, 77, 409-417, (2008).

(25) P. Feng, C. Lingfeng, Y. Hao, W. Hongjuan y Y. Jian, «Synthesis and characterization of substitutional and interstitial nitrogen-doped titanium dioxides with visible light photocatalytic activity», J. Solid State Chem., 181, 130-136, (2008)

(26) H. Diker, C. Varlikli, K. Mizrak y A. Dana, «Characterization and photocatalytic activity comparisons of $\mathrm{N}$-doped $\mathrm{nc}^{-} \mathrm{TiO}_{2}$ depending on synthetic conditions and structural differences of amine sources», Energy, 36, 1243-1254, (2011).

(27) S. Gao, Z. Xue y Y. Zhong, «Preparation and photocatalytic activity of hierarchically mesoporous-macroporous $\mathrm{TiO}_{2-x} \mathrm{~N}_{x}$ ", Appl. Catal. B, 82, 208218, (2008).

(28) S.W. Xue, X.T. Zu, W.L. Zhou, H.X. Deng , X. Xiang, L. Zhang y H. Deng, "Effects of post-thermal annealing on the optical constants of $\mathrm{ZnO}$ thin film», J. Alloys Compd., 448, 21-26, (2008).

(29) A.L. Linsebigler, G. Lu y J.T. Yates, «Photocatalysis on $\mathrm{TiO}_{2}$ surfaces: principles, mechanisms, and selected results», Chem. Rev., 95, 735-758, (1995).

(30) E. Vulliet, C. Emmelin, J.M. Chovelon, Ch. Guillard y J.M. Herrmann, "Photocatalytic degradation of sulfonylurea herbicides in aqueous $\mathrm{TiO}_{2}$ ", Appl. Catal. B, 38, 127-137, (2002).

(31) O. Levenspiel, «Interpretación de datos obtenidos en un reactor intermitente», pp. 38-82 en Ingeniería de las reacciones químicas, Limusa Wiley, México, (2006).

(32) K.V. Kumar, K. Porkodi y F. Rocha, «Langmuir-Hinshelwood kinetics - A theoretical study», Catal. Commun., 9, 82-84, (2008).

(33) J.M. Herrmann, «Heterogeneous photocatalysis: fundamentals and applications to the removal of various types of aqueous pollutants», Catal. Today, 53, 115-129, (1999).

Recibido: 19/12/2009

Aceptado: 07/09/2011 